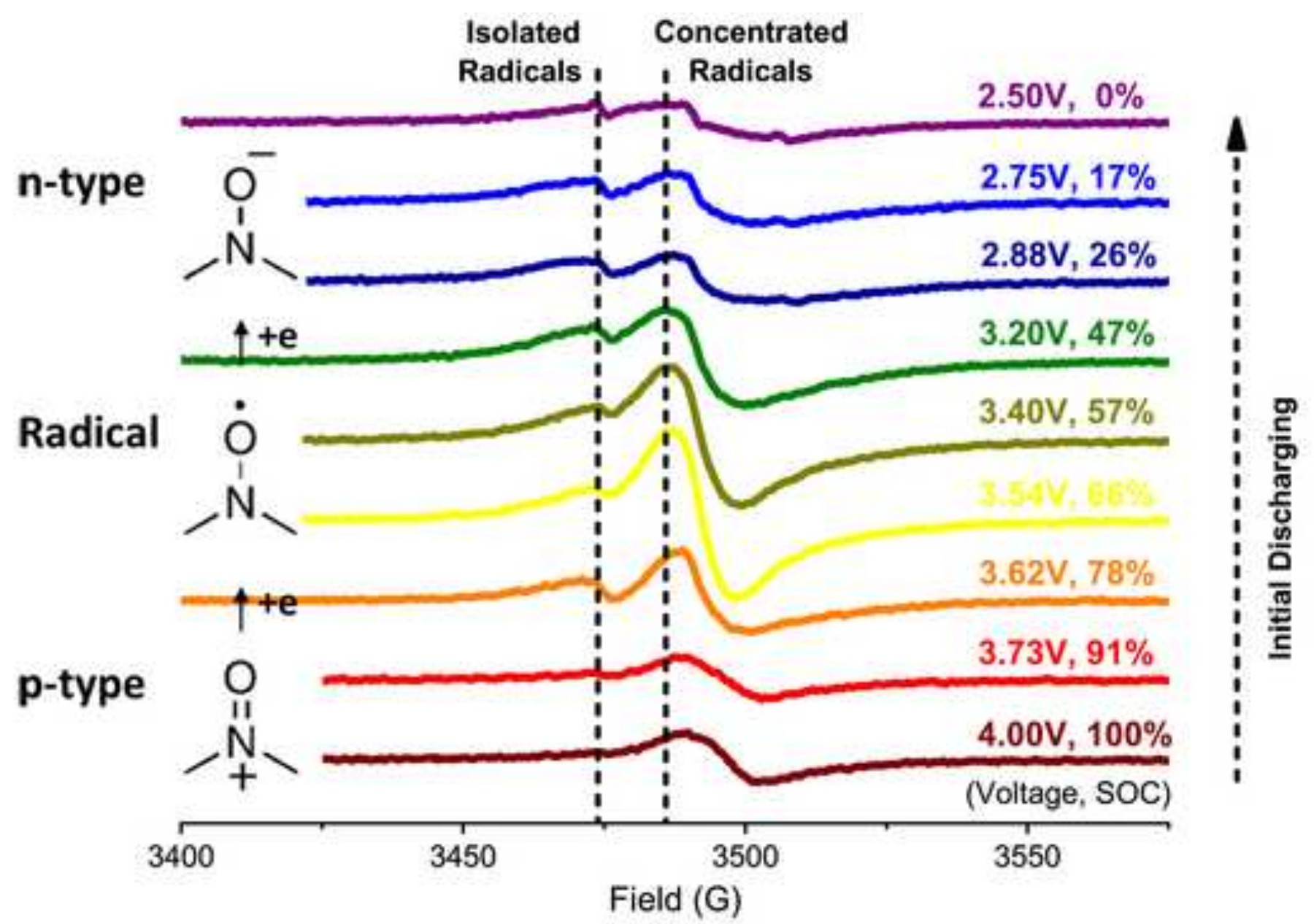




\section{In situ Electrochemical-Electron Spin Resonance Investigations of Multi-electron Redox Reaction for Organic Radical Cathodes}

Qian Huang ${ }^{a, *}$, Eric D. Walter ${ }^{\text {a }}$, Lelia Cosimbescu ${ }^{a}$, Daiwon Choi ${ }^{a}$, John P. Lemmon ${ }^{a, *}$ ${ }^{a}$ Pacific Northwest National Laboratory, 908 Battelle Boulevard, P. O. Box 999, Richland, WA, 99354, USA

* Corresponding author.

Tel.: +1 509372 6163; fax: +1 509375 4448. E-mail: Qian.Huang@pnnl.gov

Tel.: +1 509375 6967; fax: +1 509375 4448. E-mail: John.Lemmon@pnnl.gov 


\begin{abstract}
:
The multi-electron redox reaction of an organic radical based composite cathode comprised of poly(2,2,6,6- tetramethylpiperidinyloxy-4-yl methacrylate) (PTMA)-Ketjenblack is investigated using an in situ electrochemical-electron spin resonance (ESR) methodology. The experiments allow each electrochemical state to be associated with the chemical state (or environment) of the radical species upon the cell cycling. In situ ESR spectra of the composite cathode demonstrate a two-electron redox reaction of PTMA that is from an aminoxy anion (n-type, at 2.5-2.6 V vs. $\mathrm{Li} / \mathrm{Li}^{+}$) via a radical (at 3.2-3.5 V vs. $\mathrm{Li} / \mathrm{Li}^{+}$) to an oxoammonium cation (p-type, at 3.7-4.0 V vs. $\mathrm{Li} / \mathrm{Li}^{+}$). In particular, an adjustable n-type doping process of PTMA is first observed during the discharging process. Moreover, two different local environments of radical species are found in the PTMA-Ketjenblack composite electrode that includes both concentrated and isolated radicals. These two types of radical species, showing similarities during the redox reaction process while behaving quite different in the non-faradic reaction of ion sorption/desorption on the electrode surface, govern the electrochemical behavior of PTMA based composite electrode.
\end{abstract}

Keywords: organic radical cathode; lithium-ion battery; PTMA; multi-electron redox reaction; in situ electrochemical-electron spin resonance 


\section{Introduction}

The increasing necessity to develop high performance electrochemical energy storage is driven by the rapid development of portable electronic devices and electrification of transportation. State-of-art lithium-ion batteries, which have received a lot of attention [1-4], mostly use inorganic energy storage materials with the intercalation concept [5]. This charge transfer mechanism limits the capacity (140-170 mAh $\left.\mathrm{g}^{-1}\right)$, power density, safety and cycle life of batteries. Organic polymer based energy storage materials are an attractive alternative due to their inherent advantages that include: tunable redox property and high theoretical capacity, potentially high energy and power properties by varied charge transfer mechanisms, along with the added benefits from polymer materials such as environmentally-benign, light weight, and mechanic (elastic) properties [6,7]. In particular, radical polymers show promise as cathode candidates for lithium-ion batteries [8]. Poly(2,2,6,6- tetramethylpiperidinyloxy-4-yl methacrylate) (PTMA) containing a robust nitroxide radical group was first reported as organic radical cathode in $2002[9,10]$. The nitroxide radical, with a resonance structure bearing a localized unpaired electron on the nitrogen and oxygen atoms, provides high chemical stability and two redox couples [10-12]: on the anodic side, the nitroxide is oxidized to form the oxoammonium cation that corresponds to a p-type doping; and on the cathodic side, the nitroxide is reduced to the aminoxyl anion that corresponds to an $n$-type doping $[11,12]$. In the reported organic radical batteries, however, the nitroxide polymer based cathode has mostly shown one redox couple on the anodic side (p-type doping) at $3.6 \mathrm{~V} \mathrm{vs}$. $\mathrm{Li} / \mathrm{Li}^{+}$, which results in a low specific capacity of $\sim 100 \mathrm{mAh} \mathrm{g}^{-1}$ [9]. Recently an organic radical composite cathode comprised of PTMA-Ketjenblack (KB), with a two-electron redox reaction, has been developed 
in our group $[13,14]$. It was found that the performance of an organic radical electrode is strongly dependent on the conductive carbon material that plays a significant role in the electron transfer through the radical electrode during the charge-discharge process [14-18]. However, there is still a lack of mechanistic investigation on the electrochemical behavior of organic radical cathodes.

Owing to the radical polymers featuring unpaired electrons as the energy storage species in organic radical batteries, electron spin resonance (ESR) spectroscopy has been proposed to explore the charge transfer mechanism of radical polymer based active materials. A pseudo in situ electrolytic ESR measurement has been developed to probe the organic radical which was dissolved into the liquid electrolyte while a fixed potential was applied to the electrolytic cell $[10,19]$. However, an in situ ESR tool has not been reported to study organic radical batteries in which the ESR spectrum for a solid state electrode is detected during the cell cycling. In this work, we develop in situ electrochemical-ESR and show how this methodology can provide insight into the multi-electron redox reaction mechanism of PTMA-KB based composite cathode by tracking the ESR signal and the electrochemical signal of radical polymer electrode simultaneously as a function of time during charge-discharge process.

\section{Experimental}

\subsection{Preparation of PTMA-KB composite electrode}


The radical polymer PTMA, employed as the electrode-active material, was prepared according to a published approach [9] with some modifications on synthesis and purification procedures. The PTMA-KB composite electrode was prepared by a simple solvent-less electrode fabrication method [13,14]: PTMA and KB (EC-600JD, AkzoNobel Corp.) were mixed in a planetary mill followed by further milling with PTFE binder. The milled PTMA-KB-PTFE composite powder was rolled into a paper electrode, with a final weight ratio of 16.7:66.7:16.7.

\subsection{Preparation and measurements of in situ electrochemical-ESR cell}

The measurements were performed in an in situ cell (Fig. 1a) constructed in a sealed rectangular glass tube $(0.8 \mathrm{~mm} \times 8 \mathrm{~mm} \times 100 \mathrm{~mm})$. It contains a working electrode (PTMA-KB composite electrode with a surface area of $1.0 \mathrm{~cm}^{2}$ and thickness of $40 \mu \mathrm{m}$ ) and an auxiliary and reference electrode (Li metal) that was positioned as fully as possible outside of the active region of the ESR resonator (to minimize a background signal from conduction electrons on $\mathrm{Li}$ ) while keeping a short distance from the working electrode (to minimize the internal impedance of the cell). The flat cell design can facilitate a maximum ESR response with minimum microwave electric field absorption when the cell is placed in the microwave cavity of an ESR spectrometer [20]. The cell was galvanostatically cycled at a current density of $4.5 \mu \mathrm{A} \mathrm{cm}{ }^{-2}$ (1/25 C rate) with the cutoff potentials of 4.0 and $2.5 \mathrm{~V}$ vs. $\mathrm{Li} / \mathrm{Li}^{+}$for charge and discharge processes respectively. Meanwhile, the in situ ESR measurement was performed by a Bruker Elexsys 580 spectrometer fitted with a SHQE resonator. See the Supplementary data for the details in ESR measurement. 


\section{Results and discussion}

\subsection{Multi-electron redox reaction of nitroxide polymer}

The charge-discharge profiles of our developed in situ cell (Fig. 1b) display multiple voltage plateaus at 3.5-3.7 $\mathrm{V}$ and 2.9-3.2 $\mathrm{V}$ respectively, consistent with the multiple redox couples of the cyclic voltammogram in a coin cell $[13,14]$, demonstrating the multi-electron redox reaction of PTMA based composite electrode. The initial discharge capacity of the in situ cell is more than $190 \mathrm{mAh} \mathrm{g}^{-1}$ that is the most capacity delivered in a coin cell $[13,14]$. Moreover, the reversible charge-discharge curves and good capacity retention in the following cycles indicate that the in situ cell is able to output the robust electrochemical performance of an organic radical cathode that is comparable to a practical coin cell system.

The ESR spectra of PTMA-Ketjenblack composite electrode were recorded during the charging and discharging of the in situ cell (Fig. 1c). Moreover, the double integration of ESR spectrum, which responds to the total radical concentration, was plotted as a function of capacity (Fig. 2). By combination with the charge-discharge profiles, the relationship between the total radical concentration and the voltage of composite electrode is established in the cell cycling process. As shown in Fig. 2a, the experiment started from an open circuit voltage of $3.1 \mathrm{~V} \mathrm{vs.} \mathrm{Li}^{-\mathrm{Li}^{+}}$for the fresh in situ cell (labelled as A), with the radical concentration about $80 \%$ of its maximum. During the initial charging, the radical concentration increased until $3.6 \mathrm{~V}$ where it reached the maximum (B). Then the radical concentration dropped down sharply during the first charge plateau and reached the minimum (C) at $3.8 \mathrm{~V}$, followed by slight increase again when continuously charging to a top cutoff voltage of $4.0 \mathrm{~V}$ (D). Here the ESR signal is significantly 
diminished in the first charge plateau indicating the electron pairing process, i.e. radical species are oxidized to the cations (p-type doping). In the discharging process (Fig. 2b), the radical concentration began with the slight decline until $3.7 \mathrm{~V}$ where it reached the minimum $(\mathrm{E})$, and then rose dramatically during the first discharge plateau to reach the maximum $(\mathrm{F})$ at $3.5 \mathrm{~V}$. The regeneration of the radicals suggests that the cations are reduced back to the radical species. With the further discharging, the radical content went through a significant decline starting from 3.2 $\vee(G)$ and reached the sub-minimum at $2.9 \mathrm{~V}(\mathrm{H}-\mathrm{I})$ followed by the minimum at the bottom cutoff voltage of $2.5 \mathrm{~V}(\mathrm{~J})$. The elimination of the unpaired electron once again indicates that the radical species are further reduced to the anions (n-type doping).

The charge-discharge process for the subsequent cycles, as shown in Fig. S1, maintains the two main voltage plateaus at $2.9-3.2 \mathrm{~V}$ and $3.5-3.7 \mathrm{~V}$ vs. $\mathrm{Li} / \mathrm{Li}^{+}$respectively; meanwhile, the double integration of ESR spectrum shows the maximum at 3.2-3.5 $\mathrm{V}$ between two main voltage plateaus, and the minimum at $2.5-2.6 \mathrm{~V}$ and $3.7-4.0 \mathrm{~V}$ respectively in each end of voltage ranges. The in situ results demonstrate a two-electron redox reaction of PTMA that is from an aminoxy anion (reduced state, at 2.5-2.6 V) via a radical (at 3.2-3.5 V) to an oxoammonium cation (oxidized state, at 3.7-4.0 V). According to our knowledge, this is the first experiment that validates the multi-electron transfer mechanism of organic radical cathode by in situ techniques. It should be noticed that the discharge process is not as straightforward as the charge process for the PTMA-KB composite electrode. In addition to the two main plateaus at 3.5-3.7 $\mathrm{V}$ and 2.9-3.2 $\mathrm{V}$, the discharge voltage profiles exhibit an additional small plateau at 2.6-2.8 $\mathrm{V}$. Accordingly, the double integration of ESR spectrum experiences an extra decline during the 
discharging below $2.9 \mathrm{~V}$ (Fig. 2b). This phenomenon indicates that the radical species may undergo a two-stage process of n-type doping in the composite electrode, i.e. one population of radicals is reduced during the discharge plateau at 2.9-3.2 V, leading to the decrease of total radical concentration to the sub-minimum $(\mathrm{H}-\mathrm{I})$; and the remaining radicals are reduced during the plateau at 2.6-2.8 $\mathrm{V}$, resulting in the further decrease of radical concentration to the minimum (J). Moreover, the shift of n-type doping process was observed with the continual cycling. As shown in Fig. S1b, more and more radicals are inclined to experience the reduction reaction at a lower voltage of 2.6-2.8 $\mathrm{V}$ instead of a higher voltage of 2.9-3.2 $\mathrm{V}$ upon cycling. The results demonstrate an adjustable $n$-type doping process in the PTMA-Ketjenblack composite electrode. The n-type doping reaction, which has rarely been reported in organic radical batteries, might be highly sensitive to the local environment of radicals and easily shifted (probably due to the poor stability of aminoxy anions at a low voltage below $3.2 \mathrm{~V}$ vs. $\mathrm{Li} / \mathrm{Li}^{+}$). This sensitivity may also be the origin that results in differences of the maximum intensity of the double integration of ESR spectrum for the discharge versus charge process. Because the peak intensities are repeatable over cycling (see Fig. S1), total number of radicals formed must be equal in both charge and discharge processes; the lower maximum intensity for the discharge is probably the result of wider range of voltages for the n-type doping process and difference in the kinetics. That is, the reaction kinetics that controls the distribution of radical species may not be reversible between the charge and discharge process. Evidence that the product distribution is under kinetic control can be seen in Fig. S2 (Supplementary Data), where the effect of different current densities is shown. 


\subsection{Local environments of radical species in the composite electrode}

The structure of ESR spectrum can provide information on the chemical state and environments of radical species beyond the concentration of radicals. As shown in Fig. 1c, the in situ spectrum of composite electrode changed significantly upon cycling. Here the spectra A-J are associated with the corresponding positions labelled in Fig. 2. In order to correlate these spectra with the local environments of radicals, we employed the EasySpin program [21] for ESR spectrum fitting and the following procedures: i) derive a minimum number of canonical spectra; ii) identify the chemical species corresponding to these spectra; and iii) determine the relative concentration of each species during the course of the charge-discharge cycle. In Fig. 1c, spectrum A of fresh composite electrode (3.1 V) gave a broad central resonance at $3485 \mathrm{G}(\mathrm{g}=2.0058)$ with small sharp peaks at $3474 \mathrm{G}$ and $3507 \mathrm{G}$. The major feature of a broad central peak of the spectrum can easily be matched with that of pure PTMA [10], indicative of a high local concentration of unpaired electrons in the composite electrode. Fitting of this spectrum with Easyspin yields two components: the majority is from PTMA (nitroxide radicals with a large amount of exchange coupling), labeled as [I] in Fig. 3, and the remainder (less than 1\%) corresponding to the solution based highly mobile TEMPO monomer, labeled as [II] in Fig. 3. With the initial charging, the amplitude of ESR spectrum reached the maximum at $3.6 \mathrm{~V}$ (spectrum $\mathrm{B}$ ), and then declined dramatically (spectrum $\mathrm{B}^{\prime}-\mathrm{B}^{\prime \prime \prime}$ ) followed by the appearance of a new spectrum at $3.8 \mathrm{~V}$ (spectrum C). Fitting of this new spectrum shows that while it has similar $\mathrm{g}$ and $\mathrm{A}$ values (Zeeman and ${ }^{14} \mathrm{~N}$ hyperfine) to basis [II], it has a lower correlation time indicating that it is partially immobilized. This new spectrum pattern is labeled as [III], attributed to nitroxide radicals with low local concentration (few radical neighbors) but located in the composite 
electrode. When continuously charging to a top cutoff voltage of $4.0 \mathrm{~V}$, the spectral pattern shifted to a weak broad peak (spectrum D). Fitting of this spectral feature, labeled as [IV], reveals that while visually similar to [I], it has a dissimilar line width and, more importantly, $g$ value (2.0028). This spectrum reflects the electrode environment -conductive carbon, based on the ESR spectrum of KB blank electrodes (Fig. S3). During the discharging, basis [I] grew in (spectrum E-E') and became the maximum at $3.5 \mathrm{~V}$ (spectrum F). It then fell gradually (spectrum $\mathrm{F}^{\prime}-\mathrm{G}$ ) to be replaced by [III] throughout 2.9-3.2 $\mathrm{V}$ (spectrum $\mathrm{H}-\mathrm{I}$ ), followed by the decrease in size below $2.8 \mathrm{~V}$ (spectrum J). As aforementioned, the radical species are supposed to experience a two-stage process of n-type doping in the composite electrode. Here the hyperfine structure of corresponding ESR spectra provides more evidence on this process: radicals with a high local concentration mostly participate in the reaction during the discharge plateaus at 2.9-3.2 V, leading to the spectrum changing from [I] to [III]; and the remaining more isolated radicals join the reaction at a lower voltage below $2.8 \mathrm{~V}$, resulting in the further decline in size of spectrum [III].

In the following charge-discharge process, the in situ ESR spectra followed this pattern repeatedly and reversibly. In order to track these changes in a systematic way, we first decomposed a representative subset of in situ spectra (A-J) using non-negative least squares in Matlab and employing the four basis spectra [I]-[IV] derived from Easyspin fitting (See the Supplementary data for the least squares fit of ESR spectra). The results in Fig. 3 indicate that while the spectra are mostly dominated by [I], there are still a certain amount of [III] that can not be ignored throughout the course of experiment. Thus it can be inferred that there are two different local environments of radical species in the composite electrode: [I] responds to the 
highly concentrated radicals, and [III] responds to the more isolated radicals. These isolated radicals are chemically different, oxidizing and reducing at different potentials than the radicals with higher local concentration. This indicates that the difference is not merely due to sterics, which would display as a sub-population that is always slower. Note that [IV] is the background from persistent radical species in carbon blacks. Although the total radical concentration (calculated from the double integration of ESR spectrum) includes the radical signal from KB in the composite electrode, it still reflects the main trend of all nitroxide species signals (shown in Fig. 3).

To further study these two types of nitroxide radicals in the charge-discharge process, the amplitude of ESR spectrum at $3485 \mathrm{G}$ and at $3474 \mathrm{G}$ (shown as red dotted vertical lines in Fig. 1c) was plotted as a function of the capacity (\%) in Fig. S4. These field positions are maxima of [I] and [III], and while they are not completely orthogonal to each other or the other bases, they can be used to draw inferences about the chemical changes during cell cycling. As shown in Fig. S4, both $3485 \mathrm{G}$ and $3474 \mathrm{G}$ exhibit one main peak with the maximum between two main voltage plateaus and the minimum in each end of voltage ranges, similar to the functional form of double integration curve in the charge-discharge process. When comparing both curves, they are found to change in a similar way during the voltage plateaus, but behave quite differently during the sloped lines of charge-discharge voltage profile. For instance, $3485 \mathrm{G}$ shows a rapid increase (or decrease) at 3.2-3.6 V and slight increase (or decrease) at 3.8-4.0 V in the charge (or discharge) process with the sloped lines. However, $3474 \mathrm{G}$ keeps almost constant in the corresponding charge (or discharge) process. Here the sloped lines respond to the non-faradic reaction of ion sorption/desorption on the surface of composite electrode, indicating the 
electric double layer capacitance (EDLC) effect that is mostly induced by the KB network [14]. Thus the results indicate that the non-faradic reaction process of ion sorption/desorption on the electrode surface leads to the distinct changes in the local concentration of concentrated radical species, while leaving the isolated radicals nearly constant. During the charge (or discharge) process, the positive (or negative) charge gathering on the surface of electrode causes the local concentration increase (or decrease) of concentrated radical species in the electrode. Here the conductive carbon KB can not only influence the local environment of nitroxide radicals, but also differentiate these radicals by EDLC effect.

\section{Conclusions}

In summary, this work has presented fundamental insight into the charge transfer mechanism of organic radical cathode by in situ electrochemical-ESR methodologies. We have demonstrated a two-electron redox reaction of nitroxide polymer based composite electrode and different local environments of radical species existing inside the electrode which play a critical role in the electrochemical behavior of nitroxide polymer. We envisage that the design and optimization of electrode environments (conductive media) will be an important subject of ongoing exploration on organic radical batteries. And the powerful in situ electrochemical-ESR shall support the development of next generation energy storage devices.

\section{Acknowledgements}


This work was supported by the Defense Advanced Research Projects Agency (DARPA) of the U.S. Department of Defense under Contract No. hr001132084. A portion of the research was performed using the Environmental Molecular Sciences Laboratory (EMSL), a DOE Office of Science User Facility sponsored by the Office of Biological and Environmental Research and located at Pacific Northwest National Laboratory (PNNL).

\author{
References \\ [1] J.-M. Tarascon, M. Armand, Nature 414 (2001) 359. \\ [2] M.Wakihara, Mater. Sci. Eng. R 33 (2001) 109.
}

[3] B. Scrosati, J. Garche, J. Power Sources 195 (2010) 2419.

[4] Z. Yang, J. Zhang, M. C. W. Kintner-Meyer, X. Lu, D. Choi, J. P. Lemmon, J. Liu, Chem. Rev. $111(2011) 3577$.

[5] M. S. Whittingham, Chem. Rev. 104 (2004) 4271.

[6] P. Novak, K. Muller, K. S. V. Santhanam, O. Haas, Chem. Rev. 97 (1997) 207.

[7] Y. Liang, Z. Tao, J. Chen, Adv. Energy Mater. 2 (2012) 742.

[8] K. Nakahara, K. Oyaizu, H. Nishide, Chem. Lett. 40 (2011) 222.

[9] K. Nakahara, S. Iwasa, M. Satoh, Y. Morioka, J. Iriyama, M. Suguro, E. Hasegawa, Chem. Phys. Lett. 359 (2002) 351. 
[10] H. Nishide, S. Iwasa, Y.-J. Pu, T. Suga, K. Nakahara, M. Satoh, Electrochim. Acta 50 (2004) 827.

[11] E. Breuer, H.G. Aurich, A. Nielsen, Nitroenes, Nitronates and Nitroxides, John Wiley \& Sons, New York 1989.

[12] L.B. Volodarsky, V.A. Reznikov, Synthetic Chemistry of Stable Nitroxides, CRC Press, Florida 1993.

[13] Q. Huang, L. Cosimbescu, P Koech, D. Choi, J. P. Lemmon, J. Power Sources 233 (2013) 69.

[14] Q. Huang, D. Choi, L. Cosimbescu, J. P. Lemmon, Phys. Chem. Chem. Phys. 15 (2013) 20921.

[15] S. Yoshihara, H. Isozumi, M. Kasai, H. Yonehara, Y. Ando, K. Oyaizu, H. Nishide, J. Phys. Chem. B 114 (2010) 8335.

[16] C. M. Liu, J. Chenz, F. Q. Wang, B. L. Yi, Russ. J. Electrochem. 48 (2012) 1052.

[17] J.K. Kim, G. Cheruvally, J.H. Ahn, Y.G. Seo, D.S. Choi, S.H. Lee, C.E. Song, J. Ind. Eng. Chem. $14(2008) 371$.

[18] W. Guo, Y.-X. Yin, S. Xin,Y.-G. Guo, L.-J. Wan, Energy Environ. Sci. 5 (2012) 5221.

[19] H. A. López-Peña, L. S. Hernández-Muñoz, J. Cardosoa, F. J. Gonzálezb, I. Gonzáleza, C. Frontana, Electrochem. Commun. 11 (2009) 1369.

[20] I. B. Goldberg, A. J. Bard, J. Phys. Chem. 75 (1971) 3281.

[21] S. Stoll, A. Schweiger, J. Magn. Reson. 178 (2006) 42. 
(a)

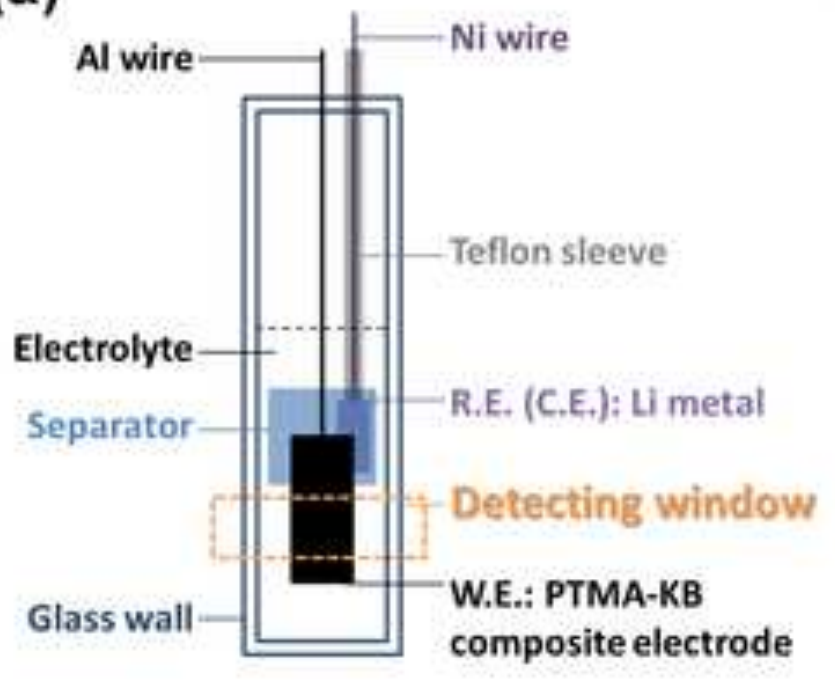

(c)
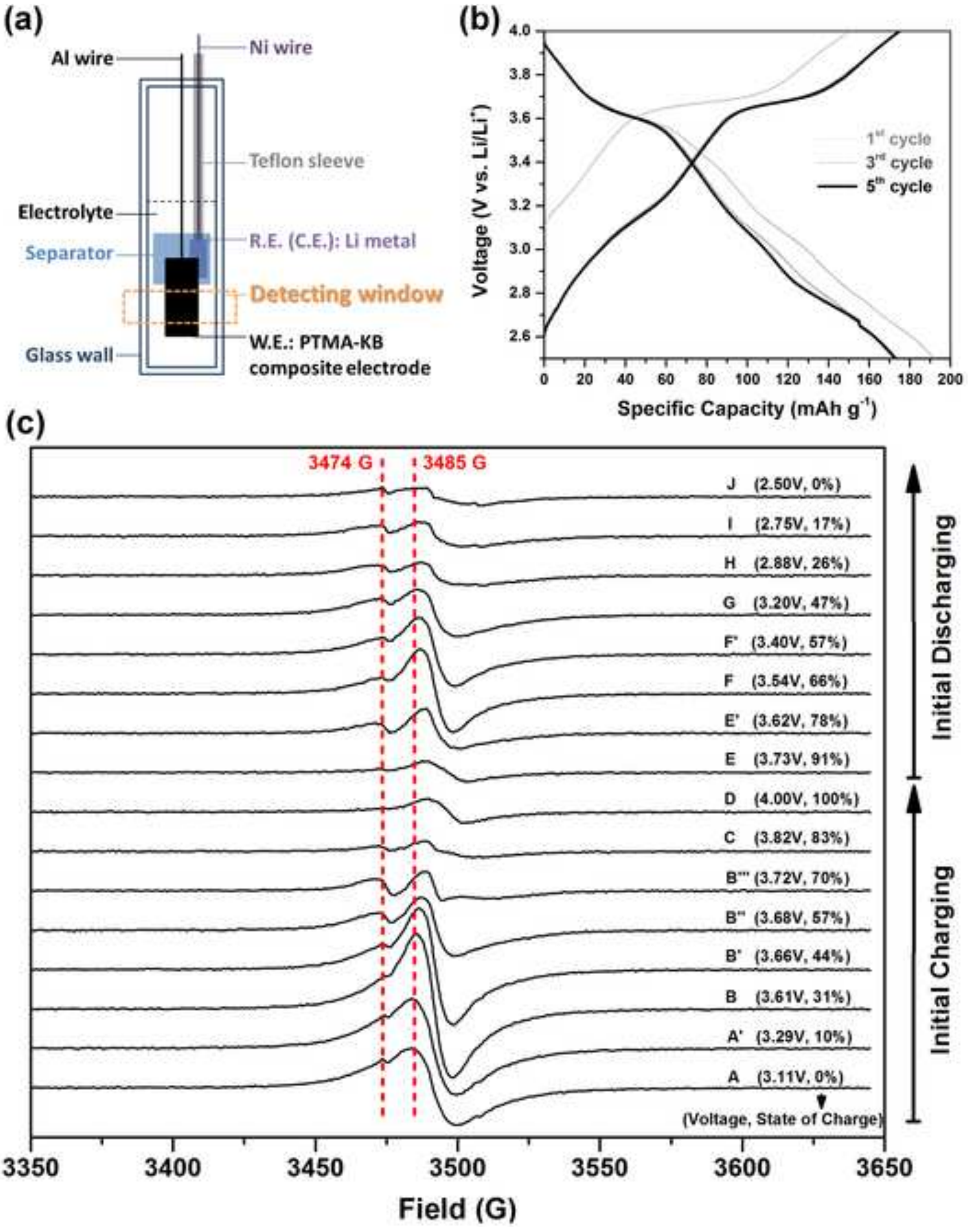
(a)

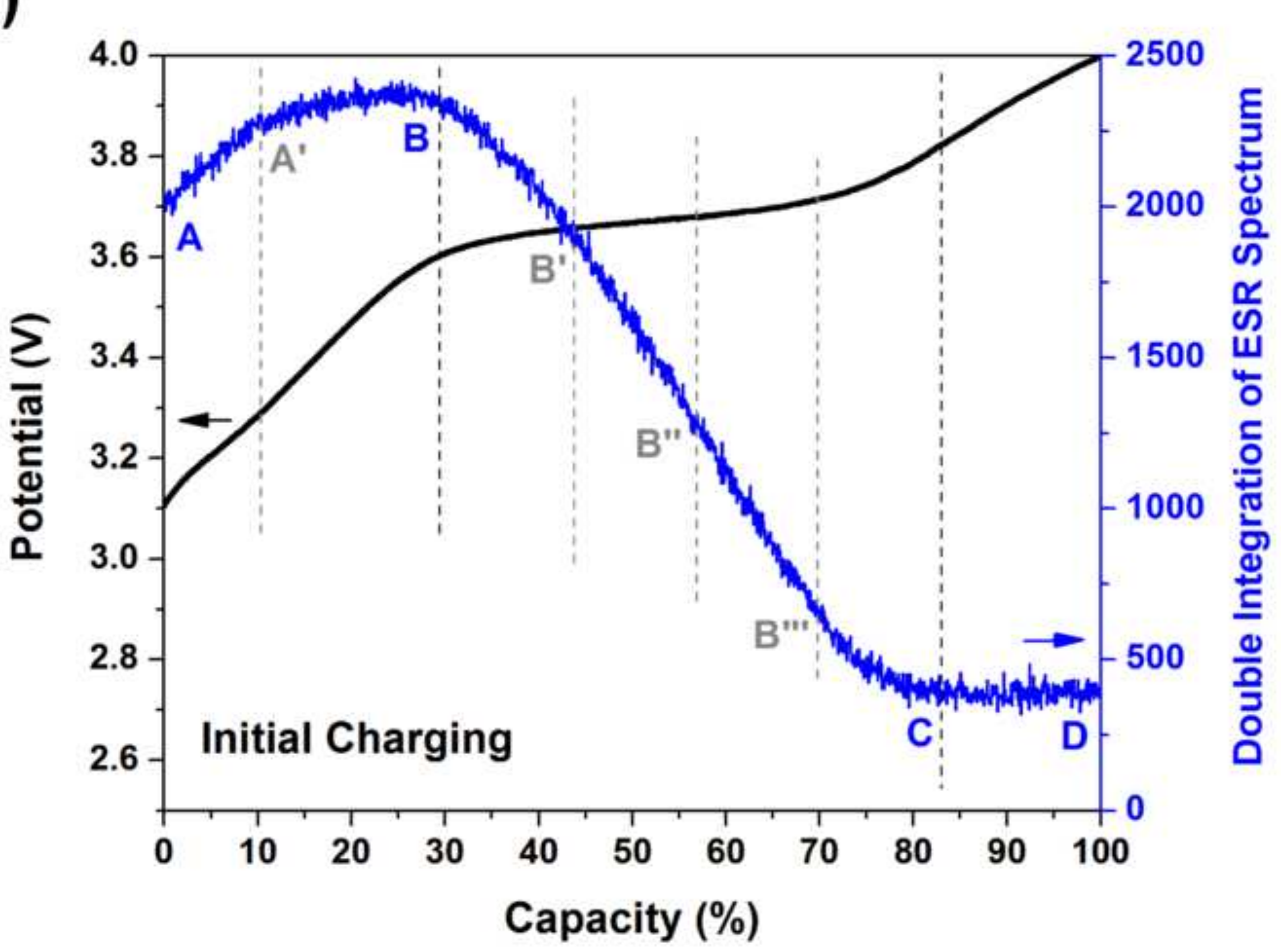


(b)

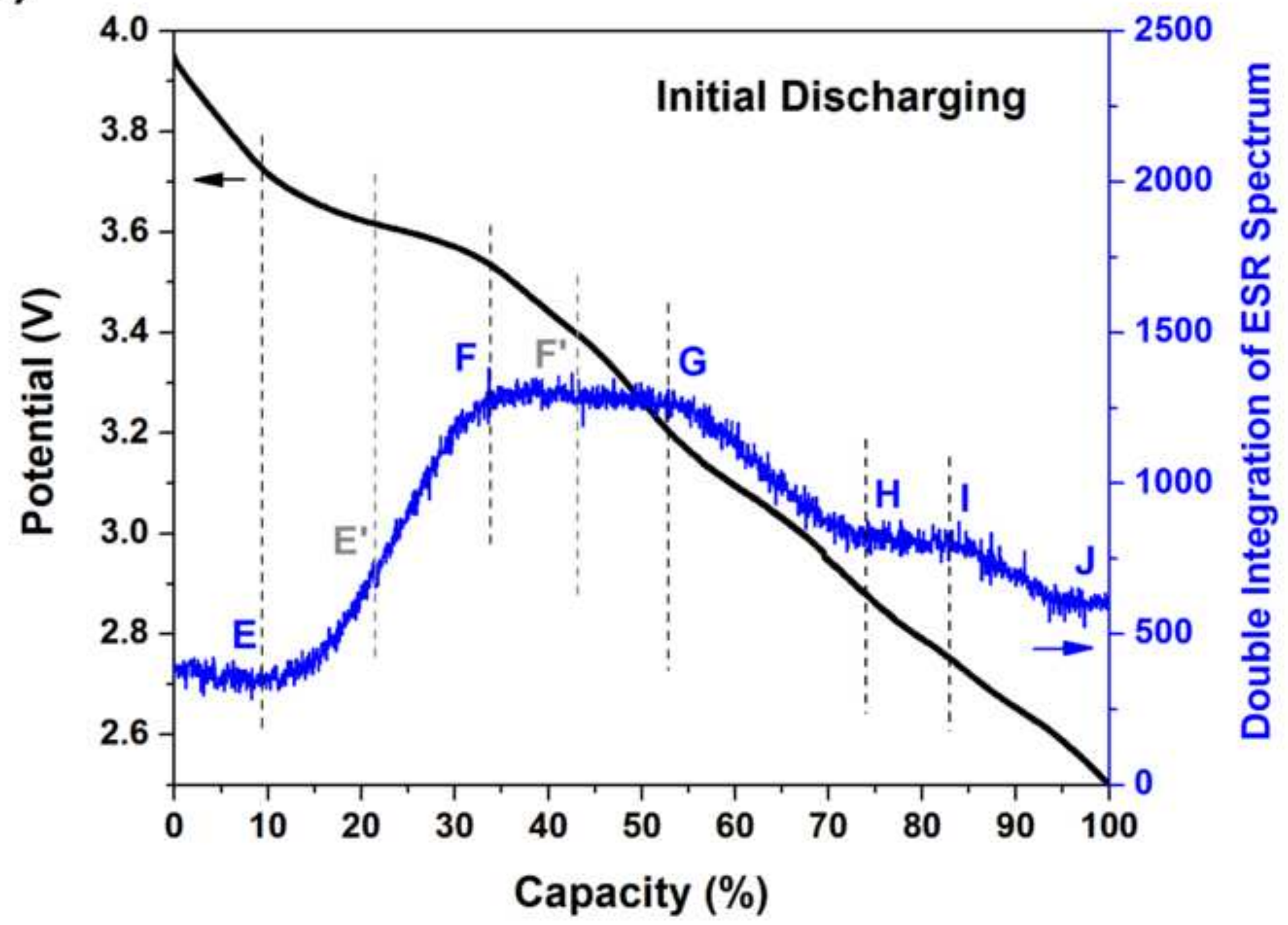




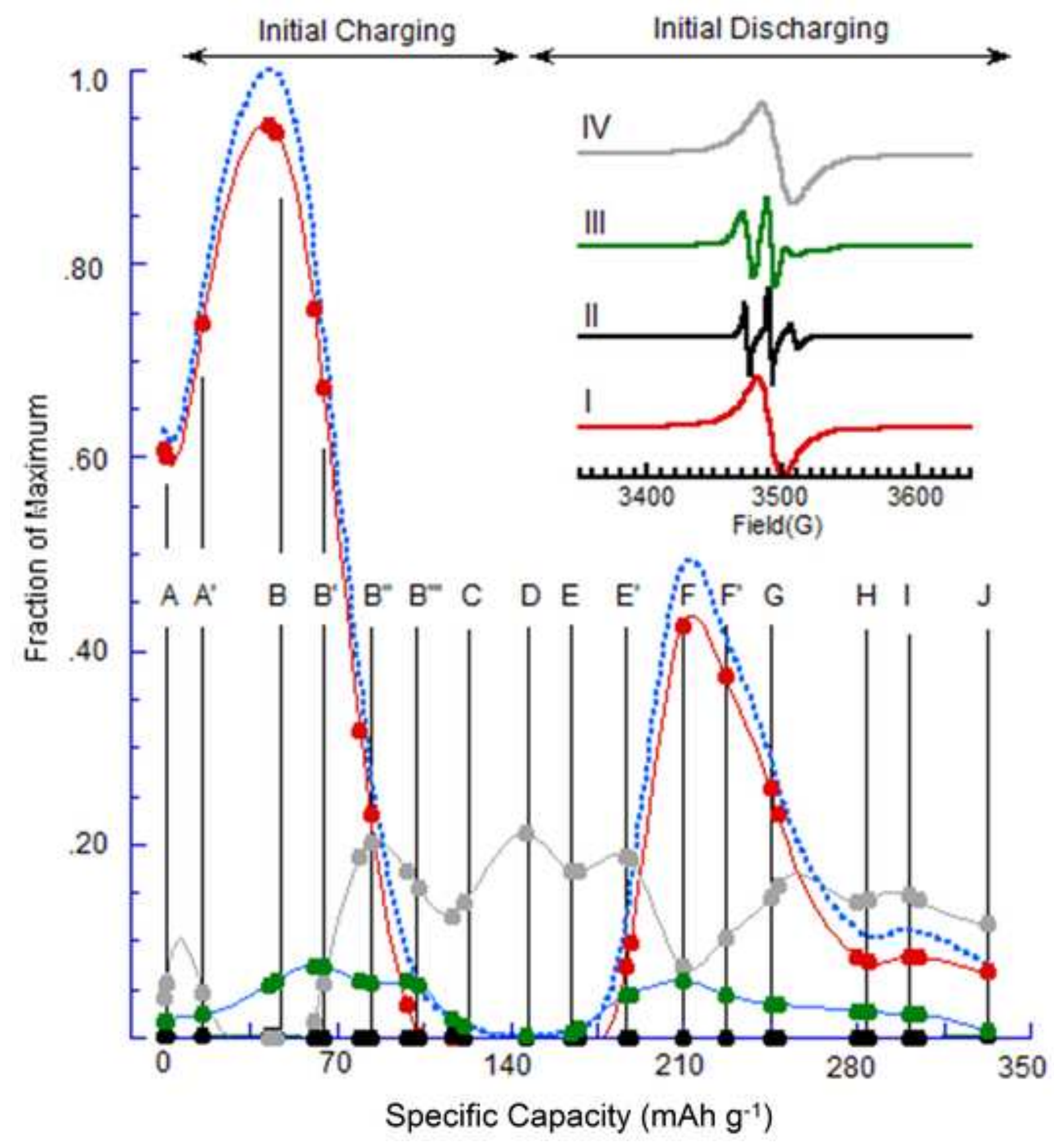

Figure 3 


\section{Figure Captions}

Fig. 1. (a) Schematic picture of the in situ electrochemical -ESR cell. (b) Charge-discharge profiles of the in situ cell at a current density of $4.5 \mu \mathrm{A} \mathrm{cm}{ }^{-2}$ between 4.0 and $2.5 \mathrm{~V}$ vs. Li/Li'. (c) Stack plot of selected ESR spectra recorded during the initial charging and discharging of the in situ cell.

Fig. 2. Double integration of ESR spectrum and voltage profile as a function of capacity (\%) for PTMAKetjenblack composite electrode based in situ cell, (a) charge and (b) discharge of the first cycle.

Fig. 3. Least Squares Fit of ESR spectra (A-J). Each spectrum was decomposed as a linear combination of four basis spectra [I]-[IV] (inset, normalized amplitude). [I] (red): nitroxides with a large amount of exchange coupling; [II] (black): highly mobile nitroxides without exchange (in solution); [III] (green): nitroxides with constrained motion; and [IV] (grey): persistent radical species in carbon blacks. Blue dashed line: the sum of [I]-[III] (all nitroxide species). 